\title{
An unusual helical micro-organism found in the gut lumen of human subjects
}

\author{
A. J. COLLINS*†, L. J. NOTARIANNI* and U. J. POTTER $\ddagger$ \\ * Department of Pharmacology, University of Bath, †Royal National Hospital for Rheumatic Diseases, Bath and \\ $\$$ Centre for Electron Optical Studies, University of Bath, Bath, Somerset BA2 7AY
}

\begin{abstract}
An earlier report described the discovery of a micro-organism in the form of a double helix in human small bowel biopsies. Mucosal biopsies of the stomach and small bowel obtained from patients with rheumatic diseases and dyspepsia by enteroscopy and gastroscopy were fixed for scanning electron microscopy to investigate the organism further. In $62 \%$ of biopsies, an organism in the form of a double helix with bifid ends, 5-30 $\mu \mathrm{m}$ long, was found lying free on the surface of the mucosa. The organism has been demonstrated in the stomach, duodenum and small bowel. Flagella were never seen to be associated with the organism. In spite of its helical form, the organism lacks many of the factors associated with spirochaete morphology. It is suggested that this, as yet unnamed organism, may be found throughout the length of the digestive tract. Its pathological significance is not known.
\end{abstract}

\section{Introduction}

A previous study demonstrated by scanning electron microscopy (SEM) that an organism in the form of a double helix, previously unknown, was present on the surface of the mucosa of human small bowel biopsies obtained at enteroscopy [1]. The organism, which appeared to be between 5 and $30 \mu \mathrm{m}$ long, was invariably seen lying on, but never invading, the small bowel mucosa. It was thought to inhabit the true small bowel rather than the duodenum. Preliminary findings suggested that the organism was present in any individual in high numbers and, although often found in patients suffering from rheumatic complaints, it was subsequently found in patients investigated for nonulcer dyspepsia who required endoscopic investigation of the upper gastrointestinal tract. This report describes further views of the organism, demonstrated by scanning electron microscopy (SEM), and suggests that it may be found not only in the true small bowel, but also in the stomach, duodenum and colon.

\section{Materials and methods}

\section{Patients}

The tissue biopsies examined in this study were taken from patients referred for endoscopic investigation of

Received 16 April; revised version received 20 July 1998; accepted 29 July 1998.

Corresponding author: Dr A. J. Collins. their gastrointestinal tract for various reasons; they were not chosen as part of a protocol. Those requiring enteroscopy [2] were suffering from forms of arthritis and were anaemic. The majority were taking nonsteroidal anti-inflammatory drugs (NSAIDs) and were suspected to be bleeding from the small bowel [3]. Biopsies of small bowel mucosa were taken as part of the investigation of the patients' anaemia, to identify lesions caused by NSAIDs and to demonstrate a normal villus pattern. Other patients requiring only gastroscopy and duodenoscopy were examined because of complaints suggestive of peptic ulcer disease. Gastric biopsies were taken to be examined for the presence of Helicobacter pylori and duodenal biopsies were taken to exclude villus atrophy. The patients were routinely prepared for endoscopic examination by being starved for at least $6 \mathrm{~h}$ before examination, and were sedated during the procedure with intravenous diazepam (Diazemuls; Dumex).

\section{Collection and preparation of gastrointestinal biopsies}

Enteroscopy was performed with an Olympus SIF-10 enteroscope (Olympus; Japan), with standard colonoscope forceps. Mucosal biopsies of the small bowel and stomach were collected by a no-touch technique. A total of 53 biopsies was taken for examination. The specimens of mucosa were washed out of the forcep cups by agitation in $5 \mathrm{ml}$ of ice-cold glutaraldehyde $2 \%$ in $0.05 \mathrm{M}$ phosphate buffer, $\mathrm{pH} 7.4$ (366 mOsms), and further processed within $48 \mathrm{~h}$ by the OTOTO method of 
post-fixation [4-6]. They were dehydrated in a graded acetone series and critical point dried from liquid $\mathrm{CO}_{2}$. Finally the specimens were sputter-coated lightly with gold and viewed in a JEOL JSM6310 SEM or a JEOL JSMT330 SEM (JEOL; Tokyo, Japan) operating at 15$20 \mathrm{KV}$.

The presence of $H$. pylori was shown by gastric mucosal biopsy. A single biopsy was tested by the direct urease test with the standard CLO test (TriMed, Carbon Court, Osbourne Park, Western Australia). Each test was read after an interval of $30 \mathrm{~min}$. No attempt was made to culture $H$. pylori from gastric biopsies to prove its presence.

\section{Results}

Examples of a double helical organism obtained from sites in the stomach, duodenum and small bowel are presented. Figs. 1-3 show examples of apparently identical organisms obtained from different sites of the gastrointestinal tract demonstrated by SEM. They were lying singly on the surface of the mucosa and in tangled groups occasionally associated with the orifices of mucus glands. They did not appear to invade the mucosal surface. The above examples are representative of the morphology of these helical organisms, which were seen to be of a regular shape and size for all the sites and various patients studied.

On an annual basis $H$. pylori is found in $18 \%$ of patients undergoing routine biopsy for upper gastro- intestinal complaints in this unit $(n>1000)$. Of the 15 gastric biopsies investigated in the present study, five were found to be positive for the presence of $H$. pylori. The presence of the helical organism did not correlate with $H$. pylori status.

\section{Discussion}

A previous report [1] described double helical organisms obtained from the human small bowel by enteroscopy and described them as spirochaetes. However, on reflection, these organisms do not readily fulfil the criteria that define the primary characteristics of spirochaetes and may represent a separate group of organisms. Characteristically, the morphology of spirochaetes includes a single protoplasmic cylinder surrounded by a gram-negative cell wall. Axial fibrils and a complex of terminal periplasmic flagella comprising an axial filament lying within an outer membrane may be present.

The double helical organisms described here have none of these primary characteristics. They vary in length from c. 5 to $30 \mu \mathrm{m}$ and are composed of a double helix, rather than a single spiral structure. The double helix appears to consist of two separate and identical spiral filaments, each filament terminating in a free fusiform end, giving the complete helix identical bifid ends. Flagella have never been seen to be associated with these organisms in any of the samples $(>50)$. Furthermore, it is generally considered that true spirochaetes do not inhabit the small bowel

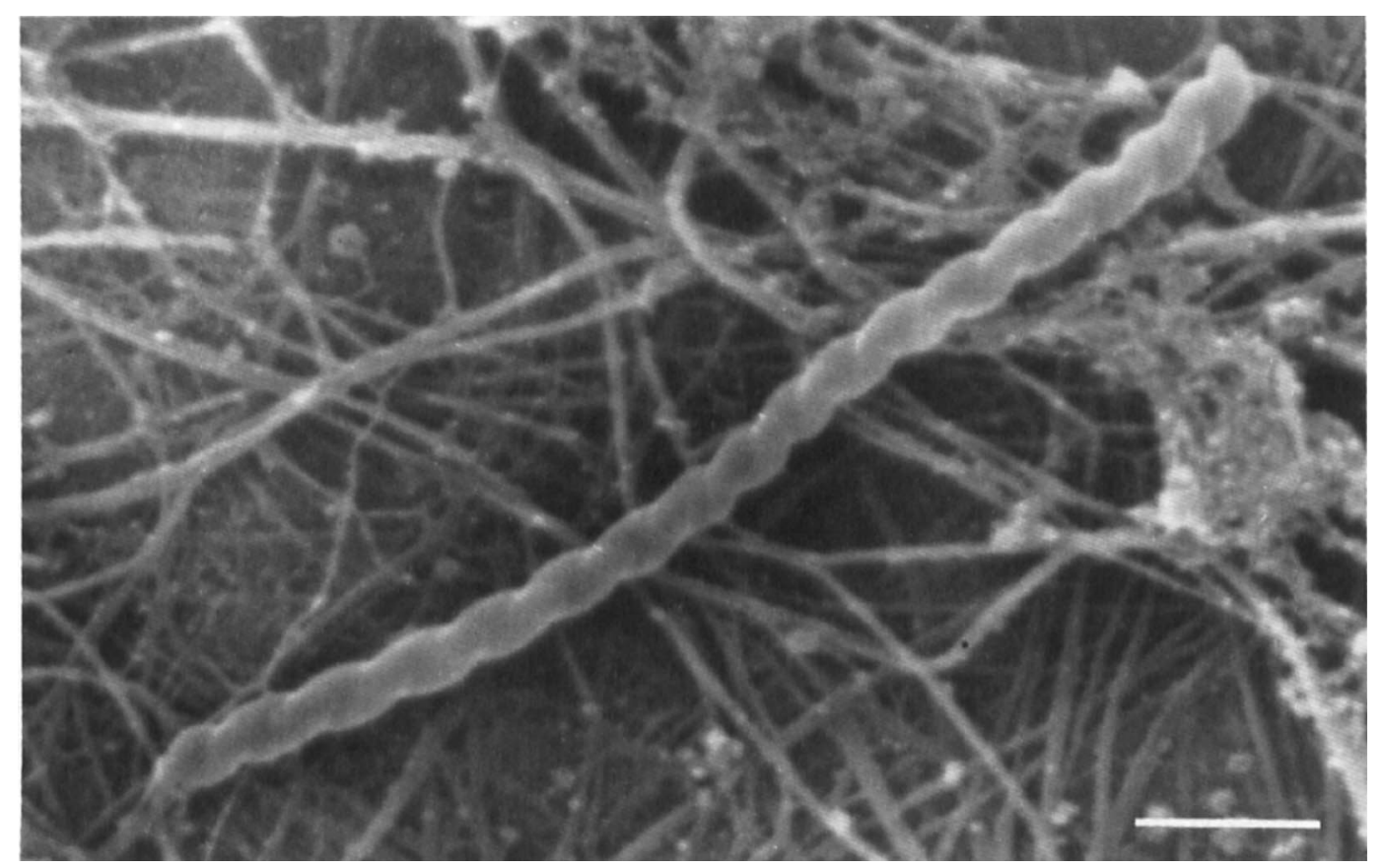

Fig. 1. Biopsy of gastric mucosa taken from the pyloric canal of an 83-year-old female patient with indigestion, who was found to have oesophagitis. The specimen was $H$. pylori negative. The filamentous background represents fixed, dehydrated gastric mucus. Lying free on the mucosal surface is a double helical organism with bifid ends. Bar $=1 \mu \mathrm{m}$. 

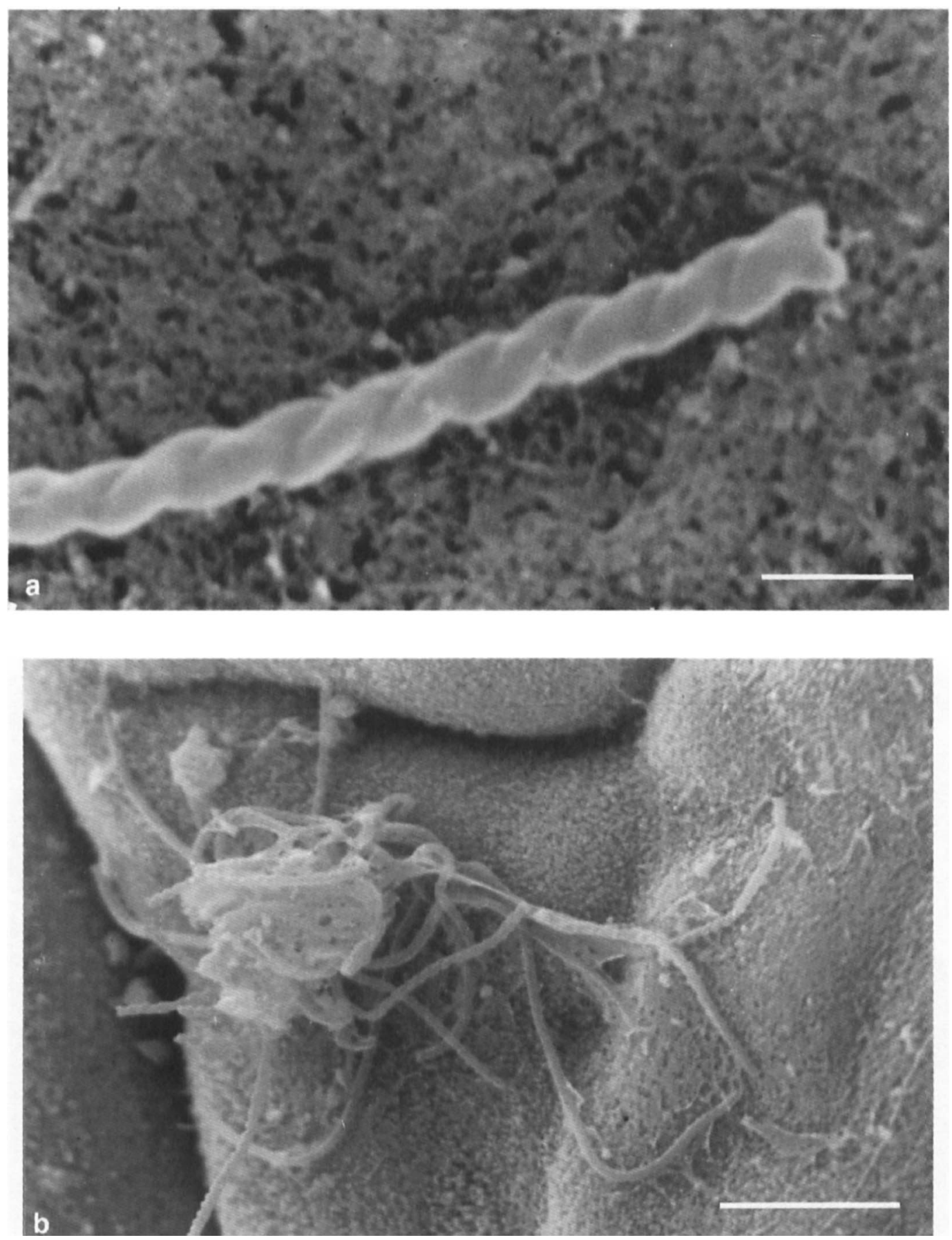

Fig. 2. Organisms from the duodenum. a, Duodenal biopsy taken from a 70-year-old female with oesophageal ulceration, bar $=0.5 \mu \mathrm{m}$. b, A tangle of organisms, mainly helical mixed with some smooth specimens, lying on the duodenal mucosa; the subject was a 60-year-old female with indigestion who was $H$. pylori negative and had an apparently normal upper gastrointestinal tract; bar $=5 \mu \mathrm{m}$.

$[7,8]$, but are commonly found in the mouth, large bowel and rectum - although one report refutes this [8].

The finding of the organism described here has been an incidental feature of the examination of biopsies taken for other reasons. No formal study of the frequency of these organisms in the gastrointestinal tract has been attempted, but it is evident from their common occurrence and the density at which they occur on mucosal biopsy specimens that they must represent frequent inhabitants of the stomach and small bowel. A review of previous studies of gastrointestinal organisms suggests that an identical organism has been demonstrated on the wall of the large bowel taken from a cadavar [9]. The results of the present study indicate that the presence of this helical organism is unrelated to a specific condition, i.e., rheumatoid arthritis or $H$. pylori status. However, the percentage of biopsies on which the organism was found in patients taking NSAIDs was $33 \%$; in other cases it ranged from 75 to $87 \%$. It must be 

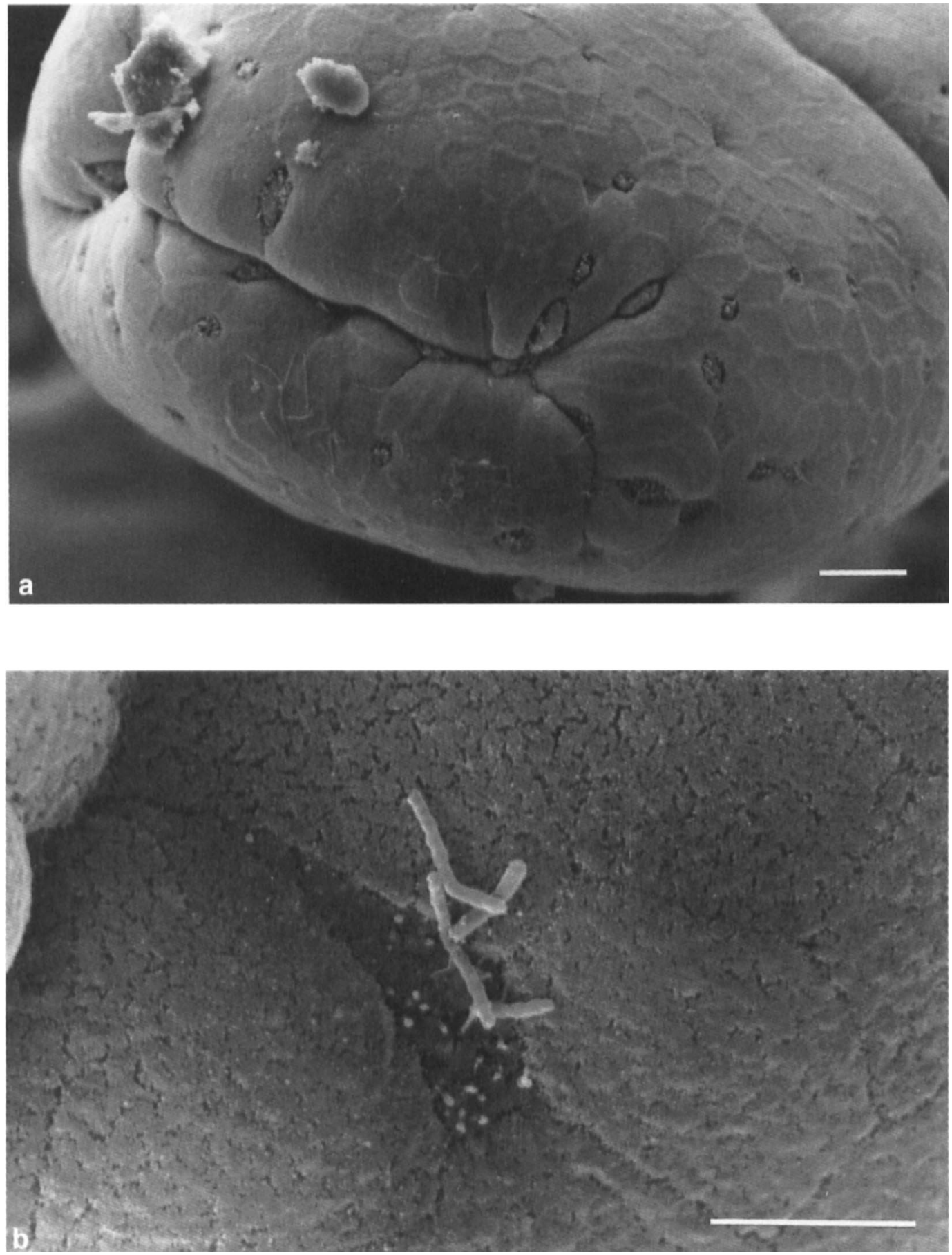

Fig. 3. Examples of helical organisms, obtained by enteroscopy from $c .60-100 \mathrm{~cm}$ distal to the pyloric sphincter in patients with rheumatoid arthritis and suspected of having associated lesions of the small bowel. a, A villus tip with terminal cleft; helical organisms lie scattered on the surface; mucus glands and individual enterocytes are apparent; bar $=10 \mu \mathrm{m}$. b. Helical organisms lying on the small bowel mucosa $100 \mathrm{~cm}$ distal to the pyloric sphincter; the outlines of individual enterocytes are visible; bar $=5 \mu \mathrm{m}$.

emphasised that these findings are not statistically sound. It is concluded that this organism, of no known pathological potential, may be found in the human gastrointestinal tract, from stomach to colon.

\section{References}

1. Potter U, Collins AJ, Notarianni LJ, Morris AJ, Smith AW. Spirochaetes in small bowel. Lancet 1995; 345: 723 .

2. Davies GR, Benson MJ, Gertner DJ, Van Someren RMN,
Rampton DS, Swain CP. Diagnostic and therapeutic push type enteroscopy in clinical use. Gut $1995 ; 37: 346-352$.

3. Morris AJ, Madhok R, Sturrock RD, Capell HA, MacKenzie JF. Enteroscopic diagnosis of small bowel ulceration in patients receiving non-steroidal anti-inflammatory drugs. Lancet 1991; 337: 520 .

4. Seligman AM, Wasserkrug HL, Hanker JS. A new staining method (OTO) for enhancing contrast of lipid-containing membranes and droplets in osmium tetroxide-fixed tissue with osmiophilic thiocarbohydrazide (TCH). J Cell Biol 1966; 30: 424-432.

5. Kelley RO, Dekker RAF, Bluemink JG. Ligand-mediated osmium binding: its application in coating biological specimens 
for scanning electron microscopy. $J$ Ultra Res 1973; 45: $254-258$.

6. Malick LE, Wilson RB. Evaluation of a modified technique for SEM examination of vertebrate specimens without evaporated metal layers. Scanning Microsc 1975; 1: 259-266.

7. Ruane PJ, Nakata MM, Reinhardt JF, George WL. Spirochete- like organisms in the human gastrointestinal tract. Rev Infect Dis 1989; 11: 184-196.

8. Les spirochètes caecaux. Ann Inst Pasteur 1927; 41: 1-44.

9. Croucher SC, Houston AP, Bayliss CE, Turner RJ. Bacterial populations associated with different regions of the human colon wall. Appl Environ Microbiol 1983; 45: 1025-1033. 